\title{
Tamaricaceae in the flora of Egypt.
}

\author{
Hasnaa A. Hosni \\ The Herbarium, Faculty of Science, \\ Cairo University, Giza - 12613, Egypt.
}

Hosni, H.A. 2000. Tamaricaceae in the flora of Egypt. Taeckholmia 20(1): 17-31.

A taxonomic revision of the family Tamaricaceae in Egypt is presented. Twelve species belonging to two genera are reported among which Tamarix chinensis Lour. is a cultivated species.

Key to the genera, sections and species based on macroscopical and microscopical characters is given. Representative specimens as well as geographical distribution and habitat for each species are also provided. Tamarix nilotica var. arabica (Bunge) H. Hosni is a new combination while Tamarix arborea (Sieb. ex Ehrenb.) Bunge and T. mannifera (Ehrenb.) Bunge are treated as distinct species.

Key words: Egypt, Tamarix, Reaumuria.

\section{Introduction}

Tamaricaceae A.St. Hill, comprises four genera and about 120 species mostly in maritime and sandy places of temperate and subtropical regions throughout the Mediterranean, $\mathrm{N}$ Africa, SE Europe and Central Asia; one species (Tamarix gallica L.) is naturalized in the New World.

The plants of this family are readily distinguished by the sinous branchlets; the appressed minute subulate leaves and the hairy seeds (Lawrence, 1951: 608).

Bentham \& Hooker (1862: 160-161), classified the family on the basis of seed characters and flower position into two main tribes: Tamarisceae and Reaumurieae; the former comprises Tamarix L., the largest genus with 90 species native to western Europe, the Mediterranean, N Africa, NE China and India; and Myricaria Desv. with 10 species in Europe and China; both are characterized by their numerous flowers in a spike like or raceme inflorescence and non endospermic seeds and comose at the apex. Reaumurieae comprises Reaumuria Hasselq. ex L. with 20 species native to E Mediterranean and C Asia; and Hololachne Ehrenb. with only 2 species, both have solitary flowers and endospermic seeds, long pilose all over (Chant, 1978: 109-110).

The family is of little economic importance, several species of Tamarix are used as wind breaks and as hedge plants especially near the sea shore; also useful dune binders and effective in fixation of river banks.

According to Chant (op. cit.: 110), the insect galls on species of Tamarix especially T. aphylla and T. gallica are a source of tannin dyes and medical extracts. The twigs of Tamarix mannifera yield a white sweet substance "Manna" as a result of puncture by the insect Coccus maniparus. The wood of Tamarix aphylla is used for house construction in N Africa and Arabia when the timber is scarce; several species of Tamarix are cultivated as ornamental shrubs.

According to Täckholm (1974: 366-369), the family is represented by 9 species related to the genera. Tamarix and Reaumuria. According to El Hadidi \& Fayed (1994/95: 101-102), and Boulos (1995: 95-96) the number of species has been raised to 10. 


\section{H.A. Hosni}

The present account aims to revise systematically the indigenous species of Tamaricaceae in Egypt with special reference to the floral and leaf characters. It is based on plant materials which were collected during field trips, also on the collections of Cairo University Herbarium (CAI) and those of the Agricultural Research Centre, Flora and Phytotaxonomy Herbarium (CAIM).

Phytogeographical subdivisions for the representative specimens are these of ElHadidi (1993); Figure (1).

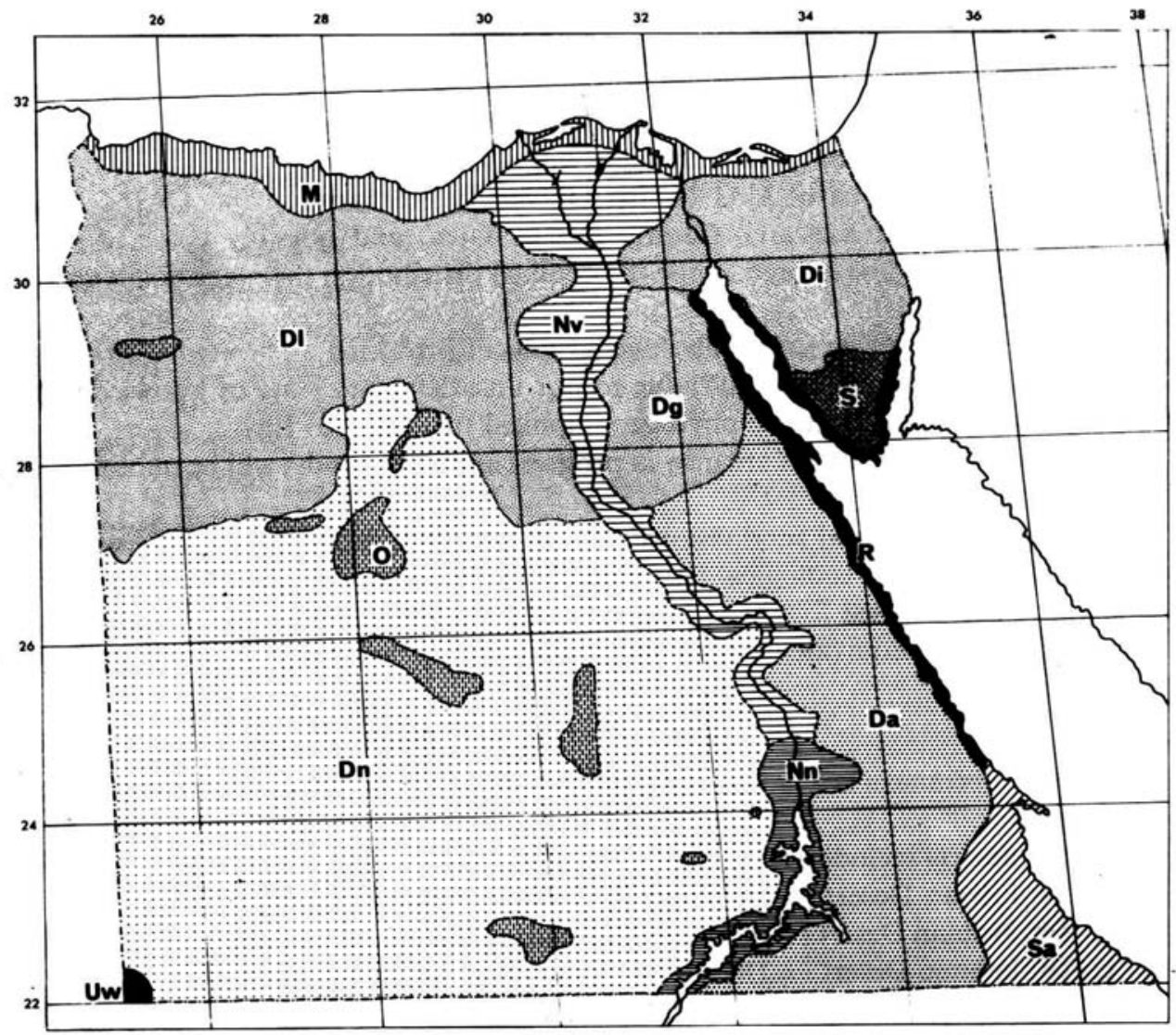

Figure (1). Phytogeographical subdivisions of Egypt (after El Hadidi, 1980). (M) Mediterranean coastal belt, (D1) Libyan Desert, (Dn) Nubian Desert, (Di) Isthmic Desert, (Dg) Galala Desert, (Da) Arabian Desert, (Nv) Nile Valley sector of the Nile-land, (Nn) Nile nubian sector of the Nile land, (O) Oases of (DI) \& (Dn), (S) Southern mountainous Sinai, (R) Red Sea coastal plains, (Sa) Gebel Elba district; (Uw) Gebel Uweinat area. 


\section{Characters of systematic value}

The characters of flower as well as the leaves provide the most reliable criteria for the delimitation of different taxa.

\section{Floral characters}

In Reaumuria species, flowers are large, up to $15 \mathrm{~mm}$ diam. subtended by an involucre of bracts. It is solitary in Reaumuria vermiculata, or in an interrupted spike in $R$. hirtella and $R$. negevensis. In Tamarix species, the flowers are small not exceeding $5 \mathrm{~mm}$ diam. subtended by a single bract and arranged in simple or compound spike-like dense racemes.

In Reaumuria stamens are numerous, \pm connate at the base in five groups inserted on a fleshy non nectariferous disc in Reaumuria species. In Tamarix, the stamens are $8-10$, distinct and diplostemonous (5 long antespalous and 5 short antepetalous) in the studied species of section Polyadenia. These are distinct and haplostemonous ( 5 fertile antesepalous stamens alternating with 5 sterile ante-petalous, each reduced into a disc-like structure) in the studied species of sections Tamarix and Oligadenia.

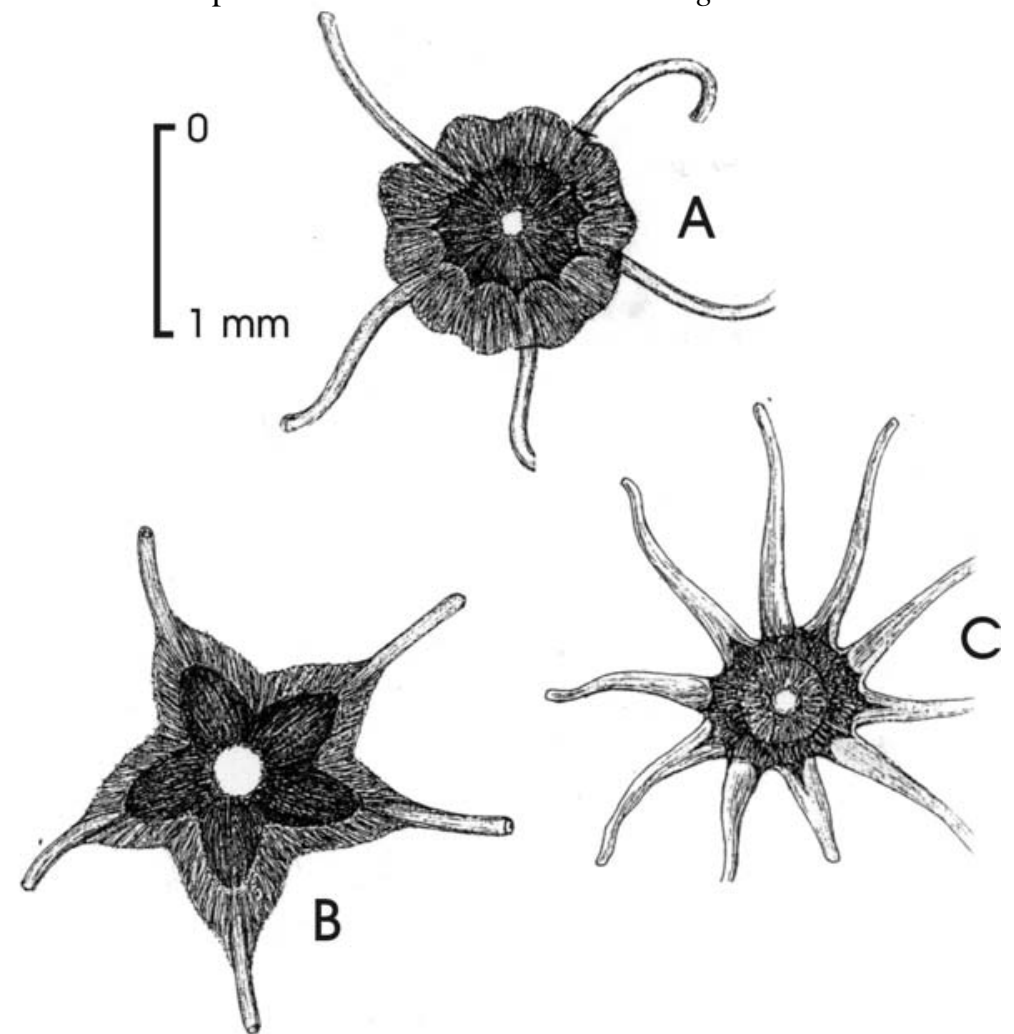

Fig. 2: Nectariferous discs in Tamarix species. A. T. nilotica var. arabica (hololophic, hypoperidiscal), B. T. arborea (synlophic, peridiscal), C. T. passerinoides (not well developed disc). 
In Tamarix nilotica var. nilotica, the filaments arise beneath the floral disc (hypodiscal); the filaments arise from the periphery of the disc (peridiscal) in T. nilotica var. microcarpa. The insertion of filaments is not constant among the examined specimens of Tamarix arabica. However, the latter taxon agrees in most of its characters with the typical Tamarix nilotica which justified its treatment in this work as var. arabica.

The nectariferous disc is not well developed among the Egyptian taxa of section Polyadenia which is the case of Tamarix passerinoides (Fig. 2, C) while the species of the sections Tamarix and Oligadenia have strongly nectariferous disc lobes. The configuration of disc can be used to distinguish certain taxa. It is for instance hololophic where the primary 5 lobes are apically distinct, among the infraspecific taxa of Tamarix nilotica. The lobes are entire with obtuse apeces in examined specimens of T. nilotica var. microcarpa as well as those of var. arabica (Fig. 2, A). The lobes are with retuse apeces in var. nilotica. It is paralophic in T. tetragyna where the lobes are deeply bipartite and each half lobe approaches the base of filaments and fused with it. In Tamarix arborea where the half lobe of the partite disc strongly units with the base of the filaments giving the impression that the filaments have a broad base (synlophic) (Fig. 2, B).

\section{Leaf characters}

Leaves are usually sessile, fleshy, dotted, conspicuously reduced or very small in Tamarix species to linear oblong in Reaumuria species.

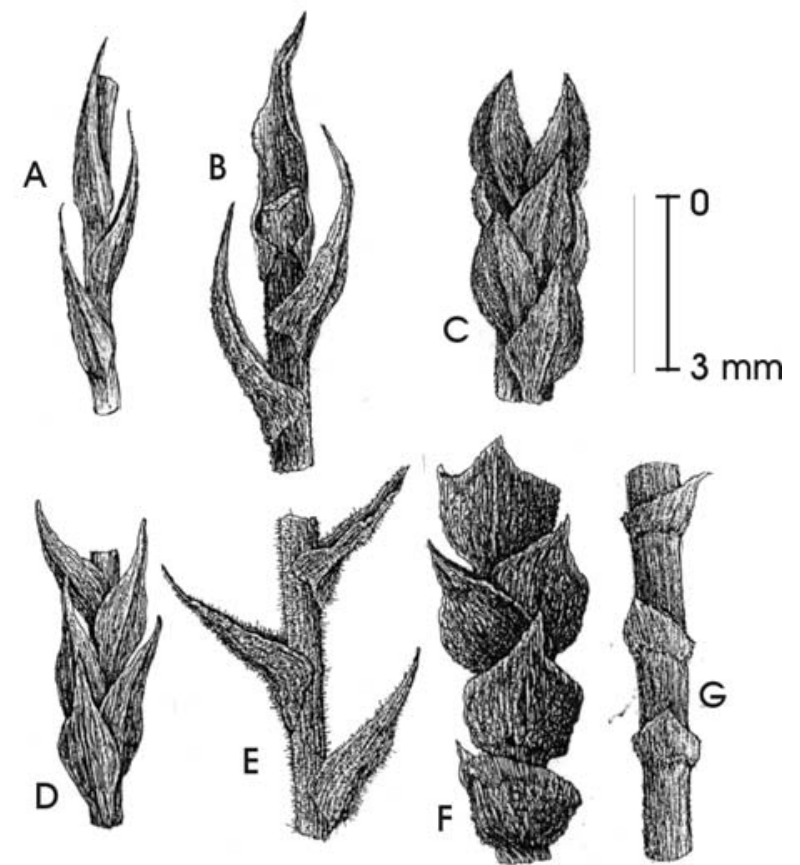

Fig. 3 : Different forms of leaves among the investigated species of Tamarix

A. T. tetragyna (sessile with narrow base), B. T. nilotica, C. T. mannifera var. arabica (sessile with auriculate base), D. T. arborea (clasping base), E. T. passerinoides (semiamplexicaul base), F. T. amplexicaulis (amplexicaule base), G. T. aphylla (reduced leaves). 
The leaf base can be used to distinguish between the species of Tamarix. It is sessile with narrow base in T. tetragyna (Fig. 3, A), sessile with auriculate base in $T$. nilotica and T. mannifera (Fig. 2, C), sessile with clasping base in T. arborea (Fig. 3, D). The leaf base is semiamplexicaul in T. passerinoides and T. macrocarpa (Fig. 3, E); strongly amplexicaul in T. amplexicaulis (Fig. 3, F) or vaginate in T. aphylla (Fig. 3, G).

\section{Synopsis to the Egyptian taxa}

\section{Tribe: Tamarisceae}

\section{Tamarix L."}

\section{Section: Tamarix}

1.1. T. nilotica (Ehernb.) Bunge

1.2. T. mannifera (Ehrenb.) Bunge

1.3. T. arborea (Sieb. ex Ehrenb.) Bunge

1.4. T. aphylla (L.) H. Karst.

Section: Oligadenia (Ehrenb.) Endl.

1.5. T. tetragyna Ehrenb.

Section: Polyadenia (Ehrenb.) Baum

1.6. T. passerinoides Delile ex Desv.

1.7. T. macrocarpa (Ehrenb.) Bunge

1.8. T. amplexicaulis Ehrenb.

Tribe: Reaumurieae

2. Reaumuria L.

2.9. $R$. vermiculata L.

2.10. R. hirtella Jaub. \& Spach

2.11. $R$. negevensis Zohary \& Danin

\section{Systematic revision}

\section{Key to the genera}

a. Shrubs or trees with flowers arranged in racemes, each flower subtended by a single bract; stamens $5-10(-12)$ free; seeds with a coma of hairs at the apex ...... 1. Tamarix

b. Dwarf shrubs with solitary flowers, each subtended by a whorl of many bracts; stamens numerous in bundles; seeds long papilose all over

2. Reaumuria

1. Tamarix L., Sp. Pl. ed. 1: 270 (1753); Gen. Pl. ed. 5: 131 (1754)

Tamariscus P. Miller, Gard. Dict., Abr. 4 (1754).

A genus of about 90 species in maritime or sandy places in W Europe, Mediterranean, through China and N Africa.

Type species: T. gallica L. (Lectotype)

The taxa are grouped according to Baum (1978) 


\section{Key to the species}

1.a. Leaves sessile with narrow base; vernal raceme (spring), 7 $10 \mathrm{~mm}$ broad; bract linear; flower tetramerous in lower part of racemes, pentamerous in upper part

\section{T. tetragyna}

b. Leaves with auriculate, semiamplexical base to vaginate; vernal racemes absent, if present $4-5 \mathrm{~mm}$ broad; bract triangular; flowers pentamerous ...... 2

2.a. Leaves auriculate or vaginate; petals $1-2.5 \mathrm{~mm}$ long, caducous; androecium haplostemonous of 5 antesepalous stamens; staminal lobes present, variously shaped

b. Leaves amplexicaul; petals 2.5 - 6 ( - 7) mm long, subpersistent, androecium diplostemonous, antesepalous stamens with long filaments, antepetalous stamens with shorter filaments, with no discal lobes

3.a. Leaves vaginate, sheath like without blade, young branches entirely glabrous

4. T. aphylla

b. Leaves narrow auriculate, auirculate or clasping; young branches papillose ... 4

4.a. Leaves auriculate or clasping, disc para-synlophic ...................... 3. T. arborea

b. Leaves with narrow subauriculate to auriculate bases; disc hololophic .......... 5

5.a. Leaves adpressed with auriculate base; sepals slightly denticulate; flower pedicel $0.5 \mathrm{~mm}$ long

2. T. mannifera

b. Leaves divaricate with subauriculate base; sepals entire; flower pedical $1-1.5 \mathrm{~mm}$ long

\section{T. nilotica}

6.a. Leaves strongly amplexicaul; flowers $2-4 \mathrm{~mm}$ diam., capsule 3 - $4 \mathrm{~mm}$ long

8. T. amplexicaulis

b. Leaves semiamplexicaul or strongly auriculate; flowers over $5 \mathrm{~mm}$ diam.; capsule (4-) 6 - 8 (-10) $\mathrm{mm}$ long

7.a. Leaves semiamplexicaul; sepals often acule \pm with entire margin; capsule ovoid - pyramidal, 2 - $4 \mathrm{~mm}$ long

6. T. passerinoides

b. Leaves strongly auriculate, outer 2 sepals often keeled, \pm obtuse with denticulate margin; capsule pyramidal, 8 - 10 mm long

7. T. macrocarpa

\section{Section: Tamarix}

Type species: T. gallica L.

1 Tamarix nilotica (Ehrenb.) Bunge, Tent. Gen. Tamar.: 54 (1852).

T. gallica L. var. nilotica Ehrenb., Linnaea 2: 269 (1827)

\section{Key to the varieties}

1.a. Bract lanceolate, $2-2.5 \mathrm{~mm}$ long, longer than the flower pedicel; the insertion of stamens is hypoperidiscal

b. Bract triangular, $1-1.5 \mathrm{~mm}$ long, as long as or shorter than the flower pedicel; the insertion of stamens is not hypoperidiscal 
2.a. Staments hypodiscal; disc lobes retuse, anthers mucronote; carpels large, $3-4 \mathrm{~mm}$ long

a. var. nilotica

b. Staments peridiscal; disc lobes obtuse, anthers apiculate; carpels small, $1.5-2 \mathrm{~mm}$ long

c. var. microcarpa

\section{a. var. nilotica}

Type: Prov. Fayum ad rivula et in insula Nili, 1820-1826; Ehrenberg (K,L, syntype)

\section{Distribution}

Common along the banks of the Nile; maritime sands of the Sinaitic sector of the Mediterranean coastal land and the Oases. Known from the Sudan, Libya, Arabian Peninsula and Palestine.

\section{Representative specimens}

M: Burg El Arab, 9.3. 1952; Helmy et al. s.n. (CAI) - EL Bouseilli, near Rosetta, 2.8. 1952; El Hadidi et al. s.n. (CAI). Port Said, 15.8.1952; Amin s.n. (CAI). DI: Qattara depression, 27.11.1967; Osborn s.n. (CAI). Di: Ismailia, El Balah road, 19.11.1979; Costantein et al. s.n. (CAI). Nv: Tahrir province, 9.7.1975; Amin et al. s.n. (CAI) - Abu Zaabal, 30.11.1951; El Hadidi s.n. (CAI) - Giza, 20.5.1926; Simpson s.n. (CAIM) - Kom Ombo, 24.12.1935; Mantasir s.n. (CAI) - Abu Simbel, 5.3.1975; El Hadidi s.n. (CAI). O: Siwa Oasis, 27.3.1927; Simpson s.n. (CAIM) - Ain Gomaa, Bahariya Oasis, 13.1.1985; Araffa s.n. (CAI) - Ain El Azzaz, Dakhla Oasis, 15.1.1998; Hosni s.n. (CAI) - Hibis Temple, Kharga Oasis, 20.6.1965; Khattab s.n. (CAIM) - Kurkur Oasis near the well, 12.12.1964; Boulos s.n. (CAI)

b. var. arabica (Bunge) H. Hosni comb. et stat. nov.

Basionym: Tamarix arabica Bunge, Tent. Gen. Tamar: 55(1852).

Type: Yemen, Arabiae Felicis in Tehama, 10.1837; Botta s.n. (holotype P, isotypes G, $\mathrm{K}, \mathrm{P}$, isotypes).

\section{Distribution}

Rare in dry wadi beds of the Eastern Desert and the Nile Valley. Known from Palestine, Jordan, Arabian Peninsula and E Africa.

\section{Representative specimens}

Di: Suez - Ismailia road, 22.9.1960; V. Täckholm et al. s.n. (CAI).

Nv: Helwan, 3.10.1952; V. Täckholm s.n. (CAI) - Kom Aushim, Faiyum, 21.9.1959; V. Täckholm s.n. (CAI). 
c. var. microcarpa (Zohary) Zohary, Fl. Palaest. 2: 329 (1972).

Basionym: Tamarix gallica L. var. microcarpa, Zohary, Trop. Woods 104:47 (1956).

Type: Palestine, Ain Hatzev, 3.3.1950; Tadmor T 1330 (HUJ, holotype).

\section{Distribution}

Known only from the coastal plains along the Red Sea. Known from Palestine.

\section{Representative specimens}

R: Red Sea Coast, between Suez \& Ain El Sokhna, 23-27.6.1960; V. Täckholm et al. s.n. (CAI).

2. Tamarix mannifera (Ehrenb.) Bunge, Tent. Gen. Tamar.: 63(1952).

Tamarix gallica L. var. mannifera Ehrenb., Linnaea 2: 270 (1827).

Type: “Egypto inferior ad Tarimon” (destroyed during world war II).

Tamarix arborea (Sieb. Ex Ehrenb.) Bunge var. mannifera (Ehrenb.) Sickenb., Mém. Inst. Egypte 4: 189 (1901).

\section{Distribution}

Salt marshes of the Mediterranean coastal land and the Oases of the Western Desert. Known from the Sudan, Palestine, eastwards to Pakistan and Afghanistan.

\section{Representative specimens}

M: Between Hamul and Baltim, 10.9.1929; M. Hefnawy s.n. (CAI). El-Arish, 15.9.1951; V. Täckholm et al. s.n. (CAI). Dg: Wadi Digla, 15.10.1953; Imam s.n. (CAI). Di: Abu Khalifa, Ballah-Ismailia, 10.8.1983; Amer 44631 (CAI)-Wadi El Arish, 7.5.1963; M.Abdallah s.n. (CAIM) - Ain El Ghedirat, 7.11.1926; Drar s.n. (CAIM). S: Wadi Feiran, 20.4.1962; Abdallah et al s.n. (CAIM).

3. Tamarix arborea (Sieb. ex Ehrenb.) Bunge, Tent. Gen. Tamar.: 67 (1952).

Tamarix gallica L. var. arborea Sieb. ex Ehrenb., Linnaea 2: 269 (1827).

Type: ad Cairo Aegypti, Sieber s.n. (PRC lectotype, G, K, OXF, P, PRC, W isolectotypes).

T. arborea var. subvelutina Boiss., Fl. Orient. 1: 776 (1867).

T. arborea var. fluviatitis Sickenb., Mém Inst. Egypte 4: 189 (1901). 
Tamaricaceae in the flora of Egypt

\section{Distribution}

Sandy and saline habitats of the Eastern Desert, along the banks of the Nile and irrigated canals as well as the Oases. Known from Libya, the Sudan and Palestine.

\section{Representative specimens}

M: Lake Burullus, 15.9.1928; Mustafa s.n. (CAI); Between Ras El Bar and Damietta, 13.9.1929; G. Täckholm s.n. (CAI); Wadi El Arish, 3.4.1988; Hosni s.n. (CAI). Di: S of El Giza pyramids, 1.1.1927; G. Täckholm s.n. (CAI). El Feirdan, Ismailia, 20.3.1974; Costantin et al. s.n. (CAI). Fayed, 13.6.1976; Simpson s.n. (CAIM) - Ain Salama, 17.1.1988; S. Araffa s.n. (CAI). Dg: N of Suez, 4.4.1927; G. Täckholm s.n. (CAI). Wadi Qosseib, 9.12.1928; Shabetai s.n. (CAI). Nv: Tell El Kebir, 7.3.1988; Amer s.n. (CAI) Beni Suef, 3.11.1928; Shabetai s.n. (CAIM). O: Siwa Oasis, near the rest, 14.4.1971; Abbas et al. s.n. (CAIM) - Bahariya Oasis, Ain Gomaa, 14.1.1988; Araffa s.n. (CAI). Dakhla Oasis, 17.1.1929; Shabetai s.n. (CAIM) - Kharga Oasis, 15.1.1928; G. Täckholm s.n. (CAI). R: Km 25 S of Hurghada, 13.9.1960; V. Täckholm s.n. (CAI). Hurghada Safaga road, 22.1.1990; Batanouny s.n. (CAI).

\section{Tamarix aphylla (L.) H. Karst., Deut. Fl.: 641 (1882).}

Thuja aphylla L., Cent. Pl. 1: 135 (1755).

Type: Egypt, specimen no. 1136/3 (LINN. , UPS, holotype).

Tamarix orientalis Forssk., Fl. Aegypt - Arab.: 206 (1775).

T. auriculata Vahl, Symb. Bot. 2: 48 (1791), nom. Illegit.

\section{Distribution}

Common in Mediterranean coastal land, Sinai, Eastern and Weatern Desert including the Oases and along the canal banks of the Nile Valley. Known from Ethiopia, Kenya, Palestine, Arabian peninsula, Iran, Pakistan and India.

\section{Representative specimens}

M: Behig, Maruit, 4.4.1935; A. Khattab s.n. (CAIM) - Amriya, 29.8.1960; Khattab s.n. (CAIM). Di: Nekhal, N. Sinai, 10.5.1939; Drar s.n. (CAIM). Dg: Ataqa, 5.11.1960; V. Täckholm et al. s.n. (CAI) - N Galala, 26.11.1944; Shabetai s.n. (CAIM). Da: Wadi El Assuityi, 6.12.1962; V. Täckholm et al. s.n. (CAI) - Wadi Qwna, 15.2.1961; V. Täckholm et al. s.n. (CAI). Nv: Sharquia, Shibtanga, 24.12.1931; Khattab s.n. (CAIM) Nn: Abu Simbel, 14.3.1931; M. Abdallah s.n. (CAIM). O: $11 \mathrm{Km}$ from Bawiti-Mines road, 8.3.1979; Abdel Ghani 1762 (CAI) - 40 Km before Farafra Oasis, 14.1.1988; Araffa s.n. (CAI) - El Dakhla Oasis, 15.1.1988; Araffa s.n. (CAI). S: Abu Zeneima, s.d.; Abdallah s.n. (CAIM). R: Wadi Araba, 7.2.1960; V. Täckholm et al. s.n. (CAI) - Wadi Gemal, 7.3.1967; Osbon et al. s.n. (CAI). 
Section: Oligadenia (Ehernb.) Endl.

Type species: T. laxa Willd. (Lectotype).

5. Tamarix tetragyna Ehrenb., Linnaea 2: 258 (1827).

Type: Egypt ad lacum Menzalah, 1820-1826; Ehrenberg s.n. (K, B, isotypes).

T. effusa Ehrenb., Linnaea 2: 258 (1827).

T. deserti Boiss., Diagn. Pl. Orient. 1(10): 9(1849).

T. tetragyna Ehrenb. var. deserti (Boiss.) Zohary, Fl. Palaest. 2: 359 (1972).

\section{Distribution}

Sandy Wadi beds, moist places and salt marshes of the Mediterranean coastal land, Galala Desert, Sinai, along the Nile banks and the Oasis. Known from Palestine, Syria, Libya and Cyprus.

\section{Representative specimens}

M: Sallum, Wadi Ramlah, 14.4.1934; Shabetai s.n. (CAIM) - El Busseilli, Rosetta, 17.9.1971; Abbas s.n. (CAIM). Dl: El Moghra Oasis, N of Wadi Qattara, 18.11.1988; Girgis \& Zahran s.n. (CAI). Di: Temsah lake, 18.3.1927; G. Täckholm s.n. (CAI). Dg: Helwan, 1.3.1940; Drar s.n. (CAI) - Cairo-Suez Rd., 3.3.1961; El Hadidi s.n. (CAI) Wadi Qosseib, N Galala, 9.2.1956; Imam s.n. (CAI). Nv: El Khanka, 18.3.1988; Araffa s.n. (CAI) - Kom Aushim, Fayium, 11.3.1987; Hosni s.n. (CAI). O: Siwa Oasis, 27.3.1927; Simpson s.n. (CAIM) - Bahariya Oasis, 5.3.1962; Saad s.n. (CAIM) - Kharaga Oasis, 10.2.1931; W. Oliver s.n. (CAI). S: Wadi El Sheikh, 15.3.1935; Nayal s.n. (CAI).

Note: Täckholm (1974: 367) regarded T. tetragyna as polymorphic including both of var. deserti (Boiss.) Zohary and var. meyeri (Boiss.) Boiss. Careful examination of the available Egyptian material is not justifying the treatment of this species as including two varieties.

Section: Polyadenia (Ehrenb.) Baum, Gen. Tamarix: 147 (1978).

Type species: $T$. passerinoides Delile ex Desv. (Lectotype).

6. Tamarix passerinoides Delile ex Desv., Ann. Sci. Nat. Bot. 1, 4:347 (1824).

Type: Egypt, Terrane, Redoute (MPU, syntype).

T. passerinoides Delile, Descr. Egypte, Hist. Nat.: 59 (1813). nom. nud. 


\section{Distribution}

Common in saline soils, sand dunes and desert wadi beds. Rare in sabkhas of the Oases and Isthmic Desert. Known from N Africa (Algeria, Libya), Palestine and Iran.

\section{Representative specimens}

M: $20 \mathrm{Km} \mathrm{S}$ of El Arish, 15.8.1951; V. Täckholm s.n. (CAI). Di: Bir Mazar, N Sinai, 15.8.1951; V. Täckholm s.n. (CAI). Nv: Dom El Hamra, Abu Rawash, 28.3.1958; Imam s.n. (CAI) - Mahalet El Hamra, Wadi El Natroun, 5.2.1968; Zahran et al. s.n. (CAI). O: Lake Zeituna, Siwa Oasis, 26.3.1927; Simpson s.n. (CAI).

7. Tamarix macrocarpa (Ehrenb.) Bunge, Tent. Gen. Tamar.: 79 (1852).

Type: In Deserto intre San et Salahie (Egypt), April 1820-26; Ehrenberg s.n. (K,L,P,UPS,W, isotypes).

T. passeriniodes Delile ex Desv. var. macrocarpa Ehrenb., Linnaea 2.276 (1827).

T. passeriniodes Delile ex Desv. var. typica Sickenb., Mem. Inst. Egypt 4: 190 (1901).

\section{Distribution}

Saline soils and sand dunes along the Red Sea and Oases, the Nile Valley and Isthamic Desert. Known from Libya, Syria, Palestine, Iran and Pakistan.

\section{Representative specimens}

Dl: $30 \mathrm{Km}$ S of Burm, Libyan desert, 3.4.1965; Osborn \& Helmy 3 (CAI). Di: Port Said Desert, Qantara - Ismailia road, 16.7.1952; Amin s.n. (CAI). Nv: Giza, Sakeit Mekki, 14.1.1926; Simpson 5913 (CAIM).

8. Tamarix amplexicaulis Ehrenb, Linnaea 2: 275 (1827).

Type: Egypt, destroyed during world war II.

T. pauciovulata J. Gay ex Battend \& Trabut, Fl. Algerie 1(2): 322 (1889).

T. trabutii Maire in Bull. Soc. Hist. Nat. Afr. N 22: 31 (1931).

\section{Distribution}

Rare in salt marshes and sandy depressions of the Oases and Sinai. Known from N Africa (Morocco and Libya) and Palestine. 


\section{Representative specimens}

DI: Qattara depression, 25.11.1976; Osborn s.n. (CAI). Di: Lake Temsah, 27.11.1911; Hartmann s.n. (CAI). O: 5 Km Mines - Bawiti road, Bahaiya Oasis, 24.1.1978; Abdel Ghani 58 (CAI) - El Qasr, El Dakhla Oasis, 5.3.1934; Shabetai s.n. (CAIM) - Kharga Oasis, Markaz Shebeika, V. Täckholm s.n. (CAI).

2. Reaumuria Hasselq. ex L., Syst. Nat. ed. 10: 1096, 1081 (17).

A genus of about 20 species from E Mediterranean to C Asia and Baluchistan.

Type species: $R$. vermiculata L.

\section{Key to the species}

1.a. Glabrous shrub; leaves, bracts and calyx lobes long mucronate; flowers solitary, terminal, filament not dilated ... $\quad \boldsymbol{9}$. R. vermiculata

b. Glabrous - hirsute shrub; leaves, bracts and calyx lobes obtuse; flowers in racemes, filament dilated

2.a. Glabrous plant with cylindrical leaves; calyx lobes with entire margin; filaments entire

b. Hirsute or puberulous plant with semicylindrical leaves; calyx lobes with irregularly denticulate margin; filaments dentate

9. Reaumuria vermiculata L., Syst. Nat. ed. 10, 2: 1081 (1759).

Type: Specimen no 701/1, (LINN, holotype).

R. mucronata Jaub. \& spach, Ill. Pl. Orient. 3: 57, t. 245 (1848).

\section{Distribution}

Uncommon species in maritime salt marshes and calcareous rocky ground of the Sinaitic sector of the Mediterranean coastal land. Known from N Africa (Libya, Algeria) and S Europe.

\section{Representative specimens}

M: $3 \mathrm{Km} \mathrm{W}$ of Sallum, 24.5.1963; V. Täckholm et al. s.n. (CAI) - Ras El Hekma, 14.4.1970; Abbas 191 (CAIM) - Matruuh, 24.7.1959; Ghabour s.n. (CAI) - El Alamein, 24.3.1961; V. Täckholm s.n. (CAI) - Abu Kir, 28.6.1912; Bolland 564 (CAIM). 
10. Reaumuria hirtella Jaub. \& Spach, II1. Pl. Orient. 3: 54 \& 55 Tab.: 244 (1848).

\section{Key to the varieties}
1.a. Stem, summer and winter leaves, bracts and sepals hirsute
a. var. hirtella
b. summer leaves pubescent, glandular; stem and winter leaves pubescent;
bracts and sepals pubescent puberulent
2.a. Bracts deflexed or spreading \pm as long as or longer than calyx
b. var. palaestina
b. Bracts appressed, much shorter than calyx c. var. brachylepis

\section{a. var. hirtella}

lectotype: In arenosis salsis Aegypti, Lippi (P).

\section{Distribution}

Wadi beds and gravel desert in Sinai and Galala Desert. Known from Palestine.

\section{Representative specimens}

Di: Ataqa, Wadi Aber, 15.2.1956; M. Imam et al. s.n. (CAI) - Gebel El Maghara, N sinai, 22.9.1985; El Hadidi \& El Garf s.n. (CAI). Dg: Helwan, El Saff, 5.1.1960; V. Täckholm et al. s.n. (CAI) - Wadi Qosseib, N Galala, 9.2.1956; V. Täckholm s.n. (CAI). S: Gebel Catherine, 24.4.1983; El Hadidi et al. s.n. (CAI). R: Wadi Feiran, Abu Zeituna, 24.4.1961; V. Täckholm et al. s.n. (CAI) - Ras Malaab, 16.8.1982; El Hadidi et al. s.n. (CAI) - Tor-Ras Mohamed road, 16.6.1965; Naguib et al. s.n. (CAI) - Ain El Sokhna, 15.1.1954; Boulos s.n. (CAI).

b. var. palaestina (Bosis.) Zohary \& Danin. Israel Journ. Bot. 19: 309 (1970).

R. palaestina Boiss., Diagn Pl. Orient. Ser. 1(10): 10 (1849).

Type: Inter St. Saba et Mare Mortuum; Boissier (G, holotype).

\section{Distribution}

Common in the wadi beds of Sinai, the Galala Desert; rare in westen Mediterranean coastal land. Known from Palestine and Jordan.

\section{Representative specimens}

M: Burg El Arab, 8.4.1955; Bot. Dept. Excursion s.n. (CAI). Di: Gebel El Maghara, 22.9.1988; El Hadidi \& El Garf s.n. (CAI) - Wadi Teba, 15.3.1935; Nayal s.n. (CAI) Cairo - Suez road, near Suez, 25.12.1959; V. Täckholm et al. s.n. (CAI). Dg: Wadi Liblab, 
6.3.1953; A. Abdel Fadeel s.n. (CAI) - Wadi Hamadel near Wadi Hof, 12.12.1952; s.n. (CAI) - Heliopolis, spring 1952; Sisi s.n. (CAI) - Wadi Amlug, 26.2.1964; M. Kassas s.n. (CAI) - Wadi Seyal, N Galala, 9.2.1956; V. Täckholm s.n. (CAI). S: Wadi Feiran, 10.5.1956; V. Täckholm 27 (CAI) - Wadi El Arbaein, 20.8.1982; El Hadidi et al. s.n. (CAI) - Gebel El Raba, 23.4.1983; El Hadidi s.n. (CAI) - St. Catherine, Wadi Talaa, 23.4.1983; El Hadidi s.n. (CAI).

c. var. brachylepis Zohary \& Danin, Israel Journ. Bot. 9: 309 (1970).

Type: Palestine, Negev, 2 Kms of Avdat, chalky slopes, S exposure, 1966; A. Danin 505 (HUJ, holotype).

\section{Distribution}

Common in calcareous soils of Isthmic and Galala Deserts, rare in W Mediterranean coastal land. Known from Palestine.

\section{Representative specimens}

M: 50 km from Mersa Matrouh, 8.8.1953; Boulos s.n. (CAI). Di: Wadi Ethelli, of Suez road, 8.1.1960; V. Täckholm et al. s.n. (CAI) - Wadi Hof, 22.5.1978; A. Bakry s.n. (CAI) - Wadi Dowiqa, E of Cairo, 3.12.1926; G. Täckholm s.n. (CAI) - Wadi El Hay, El Saff Desert, 20.12.1960; V. Täckholm et al. s.n. (CAI).

11. Reaumuria negevensis Zohary \& Danin. Israel Jour. Bot. 19:309 (1970).

Type: Palestine, central Negev, environs of Mizpe Roman, pebbly wadi bank, 700 n, 1963; Danin 12011 (HUJ, holotype).

\section{Distribution}

Uncommon in chalky ground of Northern Sinai (Isthmic Desert). Known fro Palestine.

Note: No specimens of $R$. negevensis are seen by the author, while Danin (1993: 172) reported the species from Gebel El Halal, Gebel El Maghara, Gebel Umm Kashiba and Gabel Igma (Northern Sinai).

\section{Acknowledgments}

The author is greatly indebted to Prof. Dr. M. N. El Hadidi, the Herbarium, Faculty of Science, Cairo University for reading through the manuscript. Thanks are to Mr. A. Soliman (B. Sc.) for drawing Figures 2 \& 3. 
Tamaricaceae in the flora of Egypt

\section{References}

Araffa, S. 1992. Taxonomic and ecological study of the species of the genus Tamarix L. in Egypt. M. Sc. Thesis, Botany Department, Cairo University (unpublished).

Baum, B.R. 1978. The genus Tamarix. Jerusalem, Israel Academy of Science and Humanities.

Bentham, G. \& Hooker, J. D. 1862. Genera Plantarum 1(1) : 160 - 161 - London.

Boulos, L. 1995. Flora of Egypt, Checklist : 95-96. Cairo, El Hadara Press.

Chant, S. R. in Heywood, V. H. (ed.). 1978. Flowering plants of the world, 109 - 110 Oxford University press.

Danin, A. 1993. Contribution to the Flora of Sinai IV. Comments on the "Annotated of the flora of Sinai (1989)" - Willdenowia 23 : 167-175.

El Hadidi, M. N. 1993. Natural vegetation in G. M. Craig (ed.). The Agriculture of Egypt:39-62. Oxford University Press.

\& Fayed, A. A. 1994/95. Materials for Excursion Flora of Egypt. Taeckholmia 15 : $101-102$.

Lawrence, G. H. 1951. Taxonomy of vascular plants : 607 - 608 - New York, Macmilan Company.

Metcalfe, C. R. 1950: Anatomy of the dicotyledons 1 : 157-158 - Oxford.

Täckholm, V. 1974. Students Flora of Egypt, ed. 2 : 366 - 369 - Cairo University, Cairo.

Zohary, M. \& Danin, A. 1970. The Genus Reaumuria in the Near East - Israel Journ. Bot. $19: 305-313$.

Willis, T. C. 1973. A dictionary of the flowering plants and ferns ed. 8; revised by Airy Shaw - Cambridge. 